\title{
CONE-9507/28-.1
}

\section{ON THE EXPANSION FOR SURFACE DISPLACEMENT IN THE NEIGHBORHOOD OF A CRACK TIP*}

\author{
L. J. Gray \\ Mathematical Sciences Section \\ Computer Science and Mathematics Division \\ Oak Ridge National Laboratory \\ Oak Ridge, Tennessee 37831 \\ G. H. Paulino \\ Dept. of Theoretical and Applied Mechanics \\ Cornell University \\ Ithaca, NY 14853
}

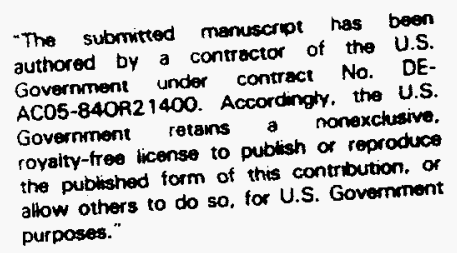

DISCLAIMER

\begin{abstract}
This report was prepared as an account of work sponsored by an agency of the United States Government. Neither the United States Government nor any agency thereof, nor any of their employees, makes any warranty, express or implied, or assumes any legal liability or responsibility for the accuracy, completeness, or usefulness of any information, apparatus, product, or process disclosed, or represents that its use would not infringe privately owned rights. Reference herein to any specific commercial product, process, or service by trade name, trademark, manufacturer, or otherwise does not necessarily constitute or imply its endorsement, recommendation, or favoring by the United States Government or any agency thereof. The views and opinions of authors expressed herein do not necessarily state or reflect those of the United States Government or any agency thereof.
\end{abstract}

* This research was sponsored by the Applied Mathematical Sciences Research Program, Office of Energy Research, U.S. Department of Energy under contract DE-AC05-84OR21400 with the Martin Marietta Energy Systems, Inc. 


\section{DISCLAIMER}

Portions of this document may be illegible in electronic image products. Images are produced from the best available original document. 


\title{
On the Expansion for Surface Displacement in the Neighborhood of a Crack Tip
}

\author{
L. J. Gray ${ }^{1}$ and Glaucio H. Paulino ${ }^{2}$
}

1 Oak Ridge National Laboratory, Oak Ridge, U.S.A.

2 Cornell University, Ithaca. U.S.A.

\section{INTRODUCTION}

For two-dimensional linear elasticity; Williams [1] has shown that the displacement field $\mathbf{u}=\left\{u_{k}\right\}, k=1.2$ in the ricinity of a crack tip is given by

$$
u_{k}(r, \theta)=a_{k}+b_{k}(\theta) r^{\frac{1}{2}}+c_{k}(\theta) r+\mathcal{O}\left(r^{\frac{3}{2}}\right),
$$

where, as illustrated by Figure 1, $r$ and $\theta$ are polar coordinates at the tip. In this figure, the mathematical crack results when the interior angle occupied by the material is $2 \pi$, i.e., $\alpha=\pi$, and the crack surfaces correspond to $\theta= \pm \pi$. In both finite and boundary element fracture analysis, the main approximation is in the

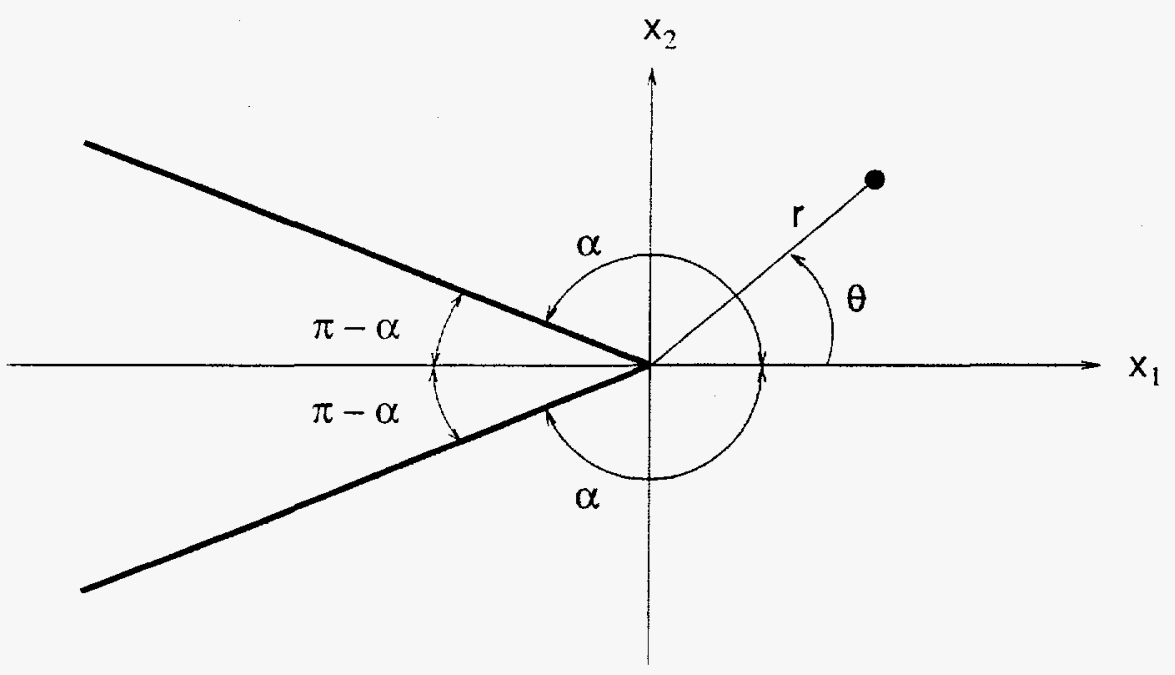

Figure 1. Definition of the coordinate systems $\left(x_{1}, x_{2}\right)$ and $(r, \theta)$ for a notch or crack geometry. The shaded portion represents the interior of the domain.

representation of the displacement. and attention has appropriately focused on capturing the $\sqrt{r}$ behavior in the approximation. This term leads to the corresponding $1 / \sqrt{r}$ singularity in the stress field, and the characterization of the fracture in terms of stress intensity factors (SIF). The 'quarter point' element $[2,3]$ is the dominant technique employed. and it is well known that use of special elements at the crack tip significantly improves the accuracy of SIF calculations [4]. 
For boundary integral fracture analysis. using either the dispiacement discontinuity method [5.6] or an approach which combines the displacement and traction boundary integral equations [7.8]. only the displacement on the crack surfaces $(\theta= \pm \pi)$. is approximated in the calculation. Thus. an appropriate near-tip crack surface interpolation of the displacement is crucial for accurate SIF calculations using these methods.

This paper points out that the expansions for the displacement on the two sides of the crack are related. in that

$$
c_{k}(\pi)=c_{k}(-\pi)
$$

Equivalently, the linear term in the jump in displacement $\Delta \mathbf{u}(r) \equiv \mathbf{u}^{+}(r)-\mathbf{u}^{-}(r)=$ $\mathbf{u}(r, \pi)-\mathbf{u}(r,-\pi)$ across the crack surface vanishes. The importance of this result, we believe, is that its inclusion in numerical approximations should improve the accuracy of the solution and the subsequent SIF evaluations. The discussion below outlines a general proof of this result in two dimensions. based upon a boundary integral formulation. The specific case of two dimensional elasticity and a traction free flat crack will be examined. A more complete discussion of Eq. (2), including a proof utilizing the eigenfunction expansion method. will be published elsewhere [9].

\section{CRACK TIP LIMITS}

The basic idea of the proof of Eq. (2) is to enforce the applied boundary condition at the crack tip. In this case, this implies that the traction must remain finite as the crack tip is approached along the crack surface. The crack tip singular

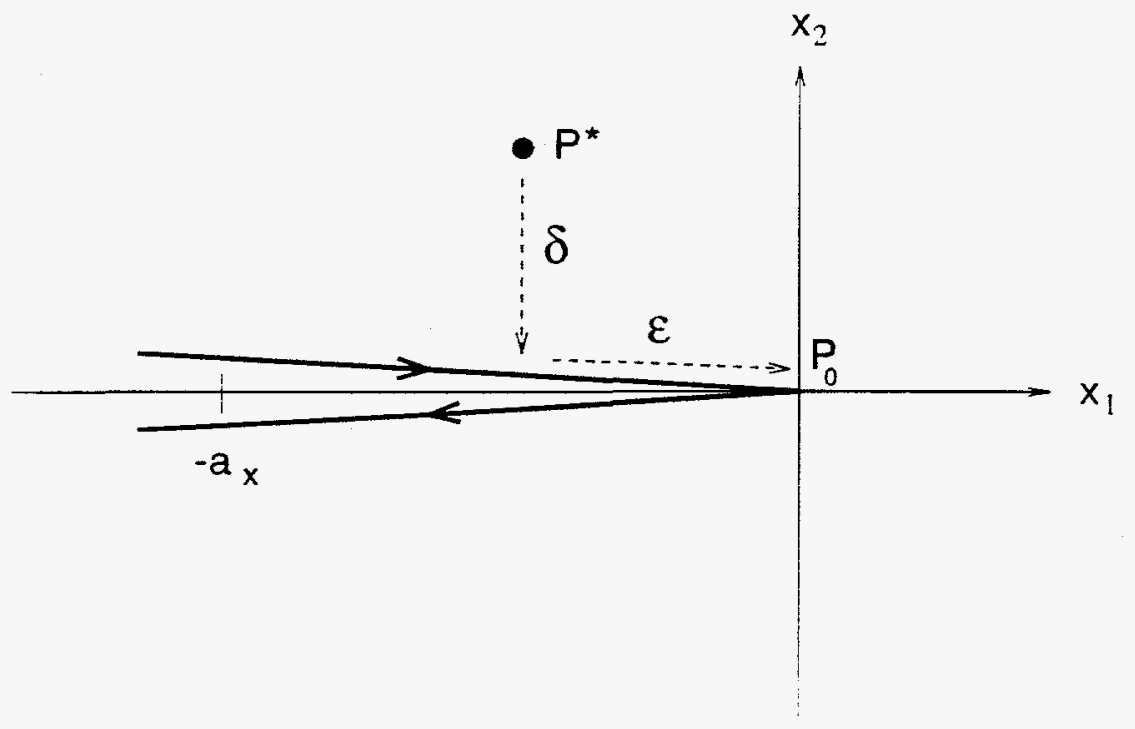

Figure 2. Illustration of the double limit process.

integrals in the hypersingular boundary integral equation for surface traction are therefore defined in terms of this limit process. Figure 2. These integrals are 

integrals are continuous crossing the boundary. Thus. the opposite orientation of the two crack tip elements ensures that the two integrals cancel. and it suffices to examine the square root and linear coefficient terms.

The crack tip element is assumed to be $\left[-a_{x}, 0\right], a_{x}>0$. parametrized as $Q(x)=\left(a_{x} x, 0\right), \quad-1 \leq x \leq 0$. Note that the integrations over the top $y=0^{+}$ and bottom $y=0^{-}$of the crack only differ by a sign, and thus it is sufficient to integrate over the top surface. Also. the traction integral (the right hand side of Eq. (3)) over the crack vanishes due to the stress-free boundary conditions. Thus, using an interior normal $\mathbf{n}=\mathbf{N}=(0,1)$ on the top crack surface $y=0^{+}$, the integral to be computed is

$$
\lim _{\varepsilon \rightarrow 0} \lim _{\delta \rightarrow 0} \int_{-1}^{0}\left(b_{m} \sqrt{x}+c_{m} x\right) S_{l k m}\left(P^{*} . Q(x)\right) d x
$$

The rather lengthy calculations are easily carried out using symbolic computation [13]. A listing of the symbolic computation programs used in this analysis can be found in Reference [9].

2.1 Square Root Mode: $u_{k}=b_{k} \sqrt{r}$

A straightforward calculation of the integral in Eq. (7) results in a complicated function of $\varepsilon$ and $\delta$. The evaluation of the limit to the boundary is accomplished by employing the Taylor expansion,

$$
\sqrt{\varepsilon^{2}+\delta^{2}}=\varepsilon\left(1+\frac{\delta^{2}}{2 \varepsilon^{2}}-\frac{\delta^{4}}{8 \varepsilon^{4}}\right)+\mathcal{O}\left(\delta^{6}\right) .
$$

Fortunately, as noted above, all of the lengthy algebra is easily handled via symbolic computation. For $\mathbf{N}=(0,1)$, the traction vector on the crack surface is $\tau=\left(\sigma_{12}, \sigma_{22}\right)$ and the calculation yields the simple result

$$
\tau=-\frac{\mu}{2 \pi(1-\nu)} a_{x}\left(\begin{array}{l}
b_{1} \\
b_{2}
\end{array}\right)
$$

The main interest of this calculation is that no potentially singular terms appear. This will not be the case for the linear term.

\subsection{Linear Mode: $u_{k}=c_{k} r$}

For this term, evaluation of the limiting value of the integral as the crack tip is approached only requires the simple observation that

$$
\lim _{\varepsilon \rightarrow 0} \lim _{\delta \rightarrow 0} \tan ^{-1}\left(\frac{\varepsilon}{\delta}\right)=\frac{\pi}{2}
$$

The potentially singular terms which arise in the evaluation of the crack tip tractions are

$$
\begin{aligned}
& \tau_{1}=\sigma_{12}=\frac{\mu}{\pi(1-\nu) a_{x}}\left(c_{1}^{+}-c_{1}^{-}\right) \log (\varepsilon) \\
& \tau_{2}=\sigma_{22}=\frac{\mu}{\pi(1-\nu) a_{x}}\left(c_{2}^{+}-c_{2}^{-}\right) \log (\varepsilon) .
\end{aligned}
$$

where $c_{k}^{ \pm}=c_{k}( \pm \pi)$. Thus. a finite ralue at the tip requires that Eq. (2) be satisfied. 


\section{CONCLLSIONS}

It has been shown that in the expansion of the crack opening displacement as a function of distance from the tip. there is no linear term present. It is hoped that exploiting this information in either finite or boundary element analyses, will lead to improved accuracy of the near tip fields, and consequently improved stress intensity factor results. Computational tests incorporating Eq. (2) are currently being implemented.

The appearance of the condition Eq. (2) and its derivation from a boundary integral formulation are not unexpected. The interpolation constraint and the method of analysis are natural extensions. to the limiting case of a crack, of previous work dealing with corner geometries [14.15]. In particular, the limit to the boundary process used to evaluate the hypersingular integrals (enforcing the traction boundary conditions on the (rack faces) is essentially the same as employed in [14]. A complete discussion of techniques for evaluating hypersingular integrals can be found in [16].

While a traction free flat crack has been treated herein. the boundary integral argument is considerably more general. The constraint, Eq. (2), holds for an arbitrary crack geometry (i.e.. multiple. non-planar), non-zero boundary conditions, and equations other than elasticity. The only assumption required is that the the form of the near tip crack surface displacement includes only a square root and a linear term, Eq. (1). Note however that even if more complicated boundary conditions or geometry (multiple, interacting cracks) should produce a term of the form $r^{\lambda}, 0<\lambda<1(\lambda \neq 1 / 2)$, it is unlikely that this will contribute a logarithmic singularity in the expression for the near tip traction. Thus, the argument leading to Eq. (2) would remain unaltered.

It is highly likely that the arguments presented here can be carried over to three dimensional crack problems. The three-dimensional computations will necessarily be more involved, but based upon previous analysis of a corner geometry [15] the extension of the limit procedure argument should be more or less straightforward. Work in this direction is currently being pursued.

\section{ACKNOWLEDGEMENTS}

This research was sponsored by the U. S. Department of Energy, Defense Programs Office of Economic Competitiveness National Information Infrastructure Major Partnership, under contract DE-AC05-84OR21400 with Martin Marietta Energy Systems. Inc. 


\section{REFERENCES}

[1] M. L. Williams. On the Stress Distribution at the Base of a Stationary Crack. AS.IE J. Appi. Mech. Vol. 24 (195T). pp. 109-114.

[2] R. S. Barsoum. On the Lise of Isoparametric Finite Elements in Linear Fracture Mechanics. Int. J. Numer. Meth. Engrg. Vol. 10 (1976). pp. 25-37.

[3] R. D. Henshell and K. G. Shaw. Crack Tip Finite Elements are Unnecessary, Int. J. Numer. Meth. Engrg., Vol. 9 (1975) pp. 495-507.

[4] G. E. Blandford, A. R. Ingraffea, and J. A. Liggett. Two-dimensional Stress Intensity Factor Computations Using the Boundary Element Method, Int. J. Num. Meth. Engrg., Vol. 17 (1981), pp. 387-404.

[5] S. Selcuk, D. S. Hurd, S. L. Crouch. and W. Gerberich, Prediction of Interfacial Crack Path: a Direct Boundary Integral Approach and Experimental Study, Int. J. Fracture, 67 (1994). pp. 1-20.

[6] T. A. Cruse. Boundary Element Analysis in Computational Fracture Mechanics, IFluwer Academic Publishers. Boston (1988).

[i] L. J. Gray, L. F. Martha. and A. R. Ingraffea. Hypersingular Integrals in Boundary Element Fracture Analysis, Int. J. Numer. Meth. Engrg., Vol. 29 (1990), pp. 1135-1158.

[8] H. K. Hong and J. T. Chen. Derivation of Integral Equations in Elasticity, J. ASCE. Eng. Mech. Div., Vol. 114 (1988), pp. 1028-1044.

[9] L. J. Gray and G. H. Paulino, Crack Tip Interpolation. Revisited, SIAM J. Appl. Math., (submitted).

[10] F. J. Rizzo, An Integral Equation Approach to Boundary Value Problems of Classical Elastostatics, Quart. Appl. Math., Vol. 25 (1967), pp. 83-95.

[11] E. D. Lutz and L. J. Gray, Exact Evaluation of Singular Boundary Integrals without CPV. Comm. Num. Meth. Engrg., Vol. 9 (1993), pp. 909-915.

[12] C. A. Brebbia, J. C. F. Telles, and L. C. Wrobel, Boundary Element Techniques. Springer-Verlag, Berlin and New York (1984).

[13] L. J. Gray, Symbolic Computation of Hypersingular Boundary Integrals, in Advances in Boundary Element Techniques. J. H. Kane, G. Maier, N. Tosaka, and S. N. Atluri, eds., Springer-Verlag, Berlin Heidelberg (1993), pp. 157-172.

[14] L. J. Gray and E. D. Lutz. On the Treatment of Corners in the Boundary Element Method, J. Comp. Appl. Math., Vol. 32 (1990), pp. 369-386.

[15] L. J. Gray and L. L. Manne. Hypersingular Boundary Integrals at a Corner, Engrg. Analy. Boundary Elem.. Vol. 11 (1993). pp. 327-334.

[16] M. Tanaka. V. Slakek. and J. Sladek, Regularization Techniques Applied to Boundary Element Methods. Applied Mechanics Reviews, Vol. 47 (1994), pp. 457499 . 